\title{
Autologous, micro-fragmented adipose tissue as a treatment for chronic shoulder pain in a wheelchair using individual with spinal cord injury: a case report
}

\author{
Chris Cherian $^{1} \cdot$ Gerard A. Malanga ${ }^{1,2,3} \cdot$ Nathan Hogaboom $\mathbb{1}^{1,4} \cdot$ Michael A. Pollack ${ }^{5} \cdot$ Trevor A. Dyson-Hudson $^{1,4}$
}

Received: 6 March 2019 / Revised: 15 April 2019 / Accepted: 18 April 2019

(c) International Spinal Cord Society 2019

\begin{abstract}
Introduction Shoulder pain is common in persons with chronic spinal cord injury (SCI), with a prevalence reported as high as $70 \%$. Current treatment of shoulder pain includes conservative measures such as physical therapy, pain medications, patient education, injections, and assistive devices. When conservative treatments fail, shoulder surgery is often the next option. Unfortunately, outcomes after shoulder surgery in persons with SCI are limited and conflicting.

Case presentation This is a case of a 54-year-old right-handed male with T10 complete SCI (duration of injury $=10$ years) who had a complaint of right-sided shoulder pain for 3 years. The individual used a manual wheelchair as his primary means of mobility and was an avid weight-lifter. Physical examination and MRI demonstrated a rotator cuff tear and degenerative changes of the acromioclavicular joint. He was previously managed conservatively with physical therapy and intermittent corticosteroid injections but failed to improve. He was enrolled in an IRB approved study and underwent an ultrasoundguided injection with autologous, micro-fragmented adipose tissue (MFAT) and ultimately received improvements in pain and function that were maintained a year after treatment.

Discussion To our knowledge, this is the first reported case of treatment of chronic refractory shoulder pain in a person with SCI using MFAT. Complete relief from pain was maintained at the 1-year follow-up. Injection of MFAT under ultrasound guidance is an effective and promising treatment for chronic refractory shoulder pain in upper limb-dependent persons with SCI and warrants further research.
\end{abstract}

\section{Introduction}

Shoulder pain is common in wheelchair using persons with spinal cord injury (SCI) [1], with a reported prevalence as high as $70 \%$ [2]. Although there are many sources of

Chris Cherian

chrischerian01@gmail.com

1 Department of Physical Medicine and Rehabilitation, Rutgers New Jersey Medical School, 183 S. Orange Avenue BHSB F-1556, Newark, NJ 07103, USA

2 New Jersey Regenerative Institute, 197 Ridgedale Ave \#210, Cedar Knolls, NJ 07927, USA

3 Kessler Institute for Rehabilitation, 1199 Pleasant Valley Way West Orange, West Orange, NJ 07052, USA

4 Kessler Foundation, 1199 Pleasant Valley Way West Orange, West Orange, NJ 07052, USA

5 Montclair Radiology, Montclair, NJ, USA shoulder pain, the rotator cuff appears to be one of the main musculoskeletal contributors [3]. Persons with SCI rely extensively on their upper limbs for many of their activities of daily living (ADLs) [2, 4]. As a consequence, chronic shoulder pain can be a major contributor to functional decline in this population [5].

Treatment of shoulder pain usually consists of conservative management with non-operative treatments (i.e. medications, physical therapy, patient education, and assistive devices) [6, 7]. If pain persists for 6 months, rotator cuff surgery is often recommended. In persons with SCI, however, the outcomes and success rates of rotator cuff surgery are equivocal [8]. Furthermore, post-surgical immobilization of the treated shoulder requires extensive rehabilitation with prolonged immobilization, switching to a power wheelchair, and assistance with weight-bearing activities of daily living $[9,10]$. Thus, alternative treatment options are needed to offer persons with SCI a way to address the pain while maintaining mobility and functional independence. 
Orthobiologics have recently been considered as an alternative treatment for various orthopedic conditions including shoulder pain. There is some evidence of the efficacy of autologous, micro-fragmented adipose tissue (MFAT) in the able-bodied population for various orthopedic conditions. Striano et al. [11] evaluated the use of MFAT for shoulder pain from rotator cuff pathology and degenerative arthritis in 18 able-bodied individuals over the course of one year. The researchers noted sustained improvement in pain, with improvement in pain on a numeric rating scale (NRS) from 7.94 to 3.7 even 12 months after the treatment. This case report highlights a successful MFAT treatment for refractory shoulder pain in a person with a chronic SCI.

\section{Case presentation}

This is a case of a 54-year-old, right-handed male with T10 complete SCI (duration of injury $=10$ years). The individual used a manual wheelchair as his primary means of mobility and was an avid weight-lifter. He had prior surgical repair of a left rotator cuff tear with ongoing symptoms. He presented with a complaint of right shoulder pain that developed 3 years prior without an obvious injury or inciting episode, with intermittent flare-ups of his pain since. His primary care physician obtained plain radiographs of the right shoulder that were unremarkable. He was then referred to an orthopedic surgeon who advised him to decrease his workout regimen and recommended conservative management of his pain. He received a corticosteroid injection (CSI) that provided temporary relief and subsequently received ongoing CSIs 2-3 times a year with short-term benefit each time. Over the past year, his pain progressed from being intermittent to constant. He rated his average shoulder pain on an 11-point NRS (where $0=$ "no pain" and $10=$ "worst pain imaginable") as 7 and noted that the pain impeded his ability to perform everyday ADLs. Over time, the individual reported diminished benefit from the CSIs he was receiving and minimal relief from using nonsteroidal anti-inflammatory medications.

\section{Physical and radiological examination}

Physical examination of the right shoulder revealed no scapular winging or shoulder muscle atrophy. Active range of motion in shoulder flexion/extension, internal/external rotation, and abduction/adduction were full, with pain noted above $90^{\circ}$ of abduction. Palpation revealed no tenderness at the bicipital groove, greater tuberosity, or acromioclavicular (AC) joint. Strength was rated as $5 / 5$ throughout the right upper extremity with an exception of pain-limited give-way in shoulder abduction and external rotation. Special maneuvers that elicited pain included positive Neer's and Yocum's tests, a painful arc, and pain with resisted external rotation.

Ultrasound examination of the right shoulder demonstrated a $0.63 \mathrm{~cm}$ articular-sided, partial-thickness tear of the supraspinatus tendon, degenerative changes of the acromioclavicular joint and posterior labrum, and mild cortical irregularity (Fig. 2a). Minimal tendinosis of the subscapularis tendon was also appreciated with no signs of supraspinatus impingement on dynamic testing. Multiplanar, multisequential MR imaging of the right shoulder was performed (3-Tesla Skyra Scanner, Siemens Medical Solutions USA, Inc., Malvern, PA) and images were evaluated by a musculoskeletal radiologist (MAP). Imaging revealed moderate, heterogenous, and increased signal intensity within the supraspinatus tendon compatible with tendinosis; severe heterogenous increased signal intensity within the infraspinatus tendon compatible with severe tendinosis; a 50 percent partial articular surface insertional tear of the anterior infraspinatus and posterior supraspinatus tendons that measured up to $10 \mathrm{~mm}$ in the transverse dimension and $12 \mathrm{~mm}$ in the anteroposterior dimension; a small amount of fluid in the subacromial/subdeltoid bursa consistent with bursitis; acromioclavicular joint arthropathy; and moderate edema within the infraspinatus muscle belly near the musculotendinous junction compatible with grade 1 strain (Fig. 1a).

\section{Treatment procedure}

The participant provided written informed consent prior to undergoing any study procedures, in accordance with procedures approved by the Institutional Review Board. Treatment involved injection of autologous MFAT under ultrasound guidance into the areas of pathology. The procedure included harvesting the lipoaspirate [12] and subsequent injection of MFAT under ultrasound guidance and is described in greater detail below.

\section{Procedure}

A 27 -gauge $\times 1.25^{\prime \prime}$ needle was used to infiltrate the skin from the lower flank, bilaterally. Adipose tissue was obtained using tumescent anesthesia of the lower flanks bilaterally. The lipoaspirate was then transferred to the Lipogems ${ }^{\circledast}$ device (Lipogems International SpA, Milan, Italy) to wash (with normal saline) and mechanically breakdown to allow injection. The final product, autologous MFAT, was then placed in $3 \mathrm{~mL}$ syringes in preparation for injection. The rotator cuff and other pertinent shoulder structures were visualized using ultrasound and then $2.5 \mathrm{~mL}$ of MFAT was injected into the supraspinatus tendon tear, $2 \mathrm{~mL}$ into the subacromial bursa, and $1 \mathrm{~mL}$ into the 


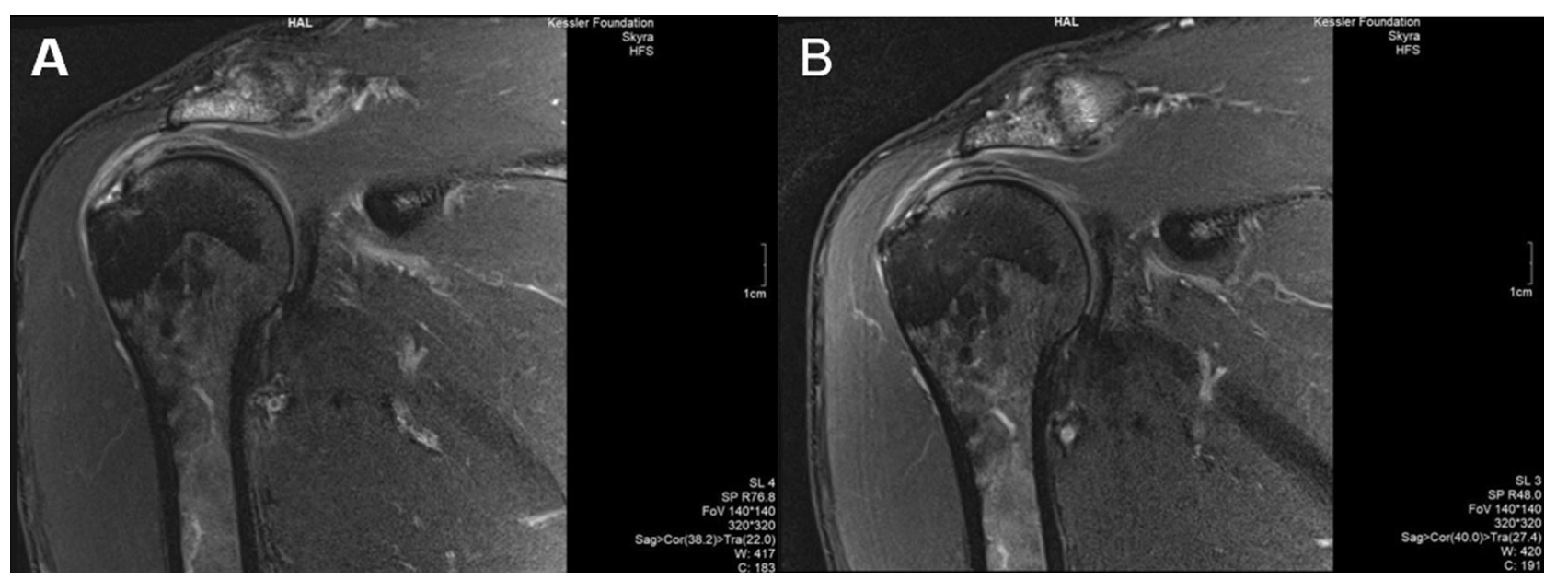

Fig. 1 Coronal T2 with Fat Saturation showing partial articular surface insertional tear of the anterior infraspinatus and posterior supraspinatus tendons prior to treatment (a) followed by improvement in partial

articular surface insertional tear of the anterior infraspinatus and posterior supraspinatus tendons 12 months post-treatment (b)

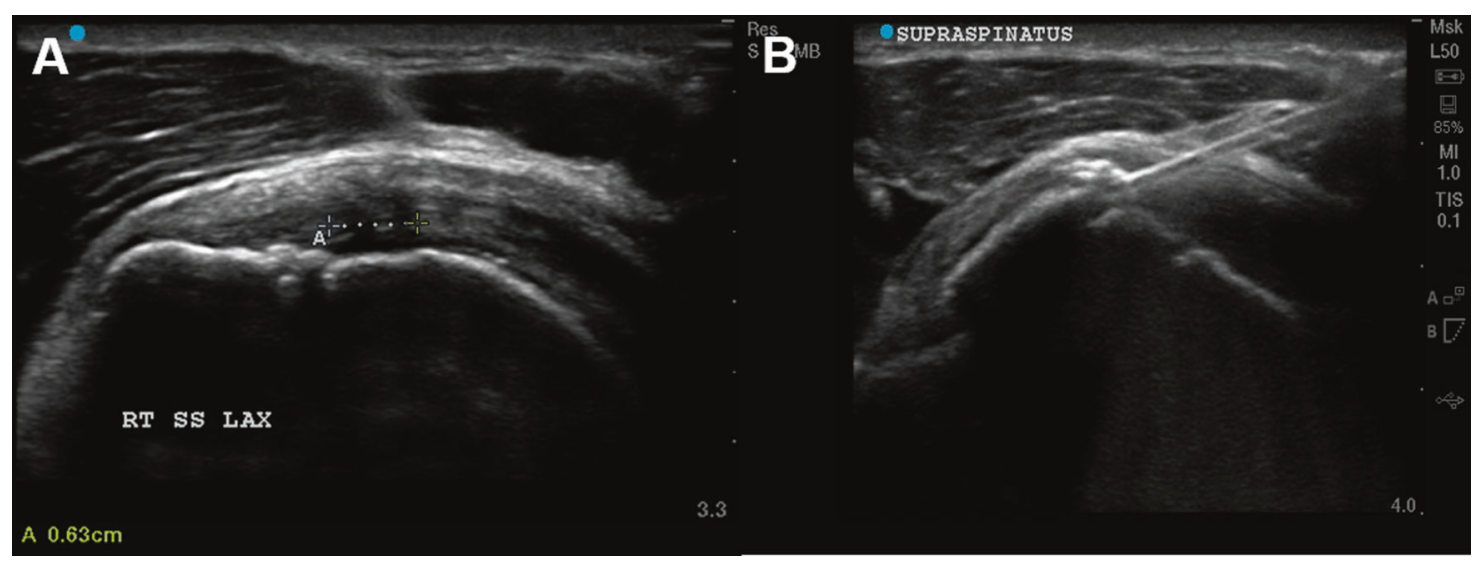

Fig. 2 Long Axis image of $0.63 \mathrm{~cm}$ articular sided tear, partial thickness tear of the supraspinatus tendon a. Long Axis image of MFAT being injected, using an in-plane approach, into the supraspinatus partial-thickness tendon tear $\mathbf{b}$

acromioclavicular joint (Fig. 2b) using an 18-gauge $\times 3.5^{\prime \prime}$ needle.

Postoperatively, participant was initially instructed to avoid upper limb weight training and to follow a period of "relative rest" for his shoulder (i.e., minimize the number of his wheelchair transfers and extended bouts of wheelchair propulsion, if possible) for the first 4 days and then to slowly increase his normal everyday upper-limb weightbearing activities, using pain as his guide. Twenty-four hours after the procedure he began the first part of a 3-part functional rehabilitation program at home. The first part involved a stretching program of the pectoralis, biceps, and trapezius muscles 4 times a day for the first month. After the first 4 weeks, the patient was instructed to perform shoulder strengthening exercises for the next 4 weeks. The exercises included scapular retractions ("rowing") with a Theraband ${ }^{\circledR}$, and shoulder abduction and shoulder external rotation with a 5-pound weight ( 3 sets of 15 repetitions every other day).
After 8 weeks, the individual was advanced to full upper limb weight-bearing activities as tolerated, including his prior workout regimen without restrictions.

\section{Treatment outcomes}

There were no adverse effects noted by the patient. Outcome measures included an 11-point numerical rating scale [13] for shoulder pain (NRS; range $0-10 ; 0=$ "no pain" and 10 = "pain as bad as you can imagine"), the Wheelchair User's Shoulder Pain Index [14] (WUSPI; range 0 to 150, with higher values representing worse shoulder pain and function), the Brief Pain Inventory interference items (BPI-I7) [15], and the Patient Global Impression of Change (PGIC) [16]. Prior to treatment, results were as follows: average, worst, and least pain NRS of 7, 10, and 3, respectively; WUSPI score of 47.6; and BPI-I7 score of 23. After the procedure, his pain lessened and he reported functional improvement. His NRS, WUSPI, 

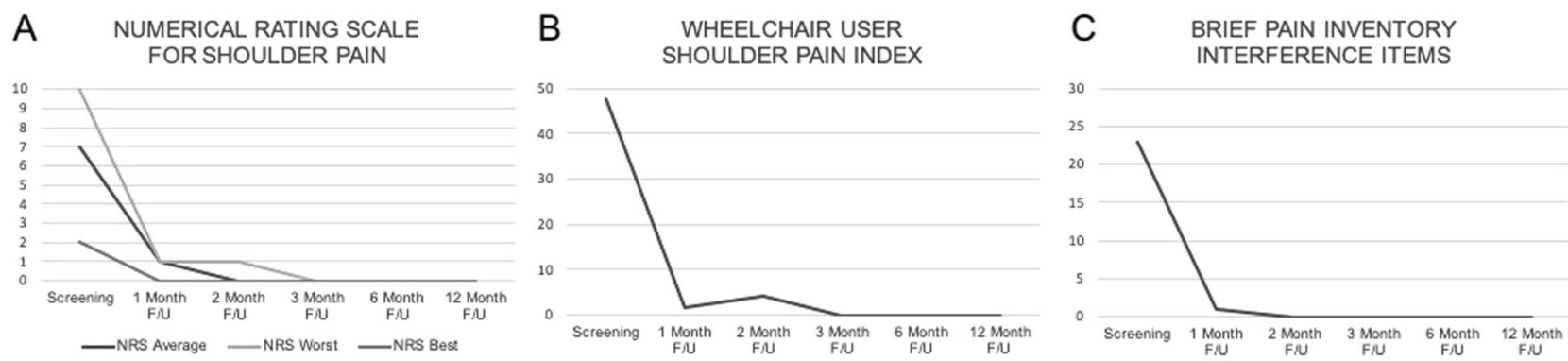

Fig. 3 Outcome measures showing improvement in Numerical Rating Scale over 12 months period a. Wheelchair User Shoulder Pain Index results for patient over 12 months period $\mathbf{b}$, followed by Brief Pain Inventory Interference Items results for the patient over the same period of time $\mathbf{c}$

and BPI-I7 steadily improved during 1, 2, 3, 6, and 12-month follow-up intervals. During his 12-month follow-up, NRS, WUSPI, and BPI-17 scores continued to be reported as 0 (Fig. $3 \mathrm{a}-\mathrm{c})$ and he reported feeling "very much improved" on his PGIC at 12 months.

Multiplanar, multisequential MR imaging of the right shoulder was repeated at 12-months post-procedure. The MR imaging revealed supraspinatus, infraspinatus, and subscapularis tendinosis with a small, partial articular-sided tear on the anterior infraspinatus tendon insertion. The tear measured $20 \%$ the thickness of the tendon $(5 \mathrm{~mm}$ in the transverse dimension and $5 \mathrm{~mm}$ in the anteroposterior dimension), which corresponded to a decrease of $50 \%$ when compared to initial assessment (Fig. 1b). The AC joint arthropathy remained unchanged and the infraspinatus muscle edema resolved.

\section{Discussion}

Shoulder pain in the SCI population is a symptom that has been noted to impair a person's functional independence [17]. Among the many causes, pain originating from rotator cuff pathology is a common cause of shoulder pain in persons who use manual wheelchairs [18]. There are limited therapeutic options for these individuals and surgical treatment is usually the next option after traditional conservative measures have failed. Unfortunately, surgical outcomes for rotator cuff repair in persons with SCI are limited and conflicting. To our knowledge, this is the first reported case of chronic shoulder pain in an individual with SCI who failed conservative management and was treated with ultrasound-guided injection of autologous MFAT. The treatment resulted in improvement of pain, function, and pathology on MRI after 12 months.

Although promising, this case report does have limitations. The results at our 12 months follow up of our patient resulted in improvements in pain and function that enabled him to return to his activities of daily living; however, there is a potential placebo effect that may have produced these subjective changes. There was also a 50 percent improvement in tear seen in the region of the posterior supraspinatus and anterior infraspinatus seen on MRI (Fig. 1b). However, the degree of tendinosis seen in the supraspinatus, infraspinatus, and subscapularis remained unchanged at 12 months. Similar outcomes have been seen with individuals undergoing regenerative procedures in the past with improvement in pain and function without significant structural changes on diagnostic imaging [19-21]. Furthermore, studies have shown that structural changes seen on MRI that may be suggestive of pathology can be seen in both symptomatic and asymptomatic patients [22]. This may suggest that the benefits of MFAT are primarily a result of its signaling and anti-inflammatory effects [23].

The MFAT injection was accompanied by needling of the tendon, which has shown some therapeutic effect for the treatment of supraspinatus tendinopathy [24]. Formal stretching and strengthening programs have also shown efficacy in wheelchair using individuals with SCI [25]. In our case, the opposite shoulder could be used as a comparator as the individual continued to have left-sided shoulder pain in spite of performing stretching and strengthening exercises in that limb as well.

Further research is needed to determine the treatment effect of ultrasound-guided injection of MFAT versus other therapies and whether this treatment should be recommended for other wheelchair users with refractory shoulder pain that is unresponsive to conservative therapy.

\section{Conclusions}

The case of refractory shoulder pain in a person with chronic SCI who was treated with ultrasound-guided injection of autologous MFAT resulting in significant improvements in pain and function and a decrease in tear size on MRI at 1-year follow-up is presented. This treatment could be a potential therapeutic option that can be efficacious in addressing chronic shoulder pain in the SCI population. 
Acknowledgements This case report is a part of a larger, IRBapproved pilot study, with funding provided for by the Derfner Foundation.

\section{Compliance with ethical standards}

Conflict of interest Gerard A. Malanga is a consultant for Lipogems ${ }^{\circledR}$. The remaining authors declare that they have no conflict of interest.

Publisher's note: Springer Nature remains neutral with regard to jurisdictional claims in published maps and institutional affiliations.

\section{References}

1. Nichols PJ, Norman PA, Ennis JR. Wheelchair user's shoulder? Shoulder pain in patients with spinal cord lesions. Scand J Rehabil Med. 1979;11:29-32.

2. Dalyan M, Cardenas DD, Gerard B. Upper extremity pain after spinal cord injury. Spinal Cord. 1999;37:191-5.

3. Bayley JC, Cochran TP, Sledge CB. The weight-bearing shoulder. The impingement syndrome in paraplegics. J bone Jt Surg Am Vol. 1987;69:676-8.

4. Curtis KA, Roach KE, Applegate EB, Amar T, Benbow CS, Genecco TD, et al. Development of the Wheelchair User's Shoulder Pain Index (WUSPI). Paraplegia. 1995;33:290-3.

5. Gerhart KA, Bergstrom E, Charlifue SW, Menter RR, Whiteneck GG. Long-term spinal cord injury: functional changes over time. Arch Phys Med Rehabil. 1993;74:1030-4.

6. Tytherleigh-Strong G, Hirahara A, Miniaci A. Rotator cuff disease. Curr Opin Rheumatol. 2001;13:135-45.

7. Van Straaten MG, Cloud BA, Zhao KD, Fortune E, Morrow MMB. Maintaining shoulder health after spinal cord injury: a guide to understanding treatments for shoulder pain. Arch Phys Med Rehabil. 2017;98:1061-3.

8. Goldstein B, Young J, Escobedo EM. Rotator cuff repairs in individuals with paraplegia. Am J Phys Med Rehabil. 1997;76:316-22.

9. Jung HJ, Sim GB, Jeon IH, Kekatpure AL, Sun JH, Chun JM. Reconstruction of rotator cuff tears in wheelchair-bound paraplegic patients. J Shoulder Elb Surg. 2015;24:601-5.

10. Kerr J, Borbas P, Meyer DC, Gerber C, Buitrago Tellez C, Wieser $\mathrm{K}$. Arthroscopic rotator cuff repair in the weight-bearing shoulder. J Shoulder Elb Surg. 2015;24:1894-9.

11. Striano RD, Malanga GA, Bilbool N, Azatullah K. Refractory shoulder pain with osteoarthritis, and rotator cuff tear, treated with micro fragmented adipose tissue. Orthop Spine Sports Med. 2018;2:014.
12. Bianchi F, Maioli M, Leonardi E, Olivi E, Pasquinelli G, Valente $\mathrm{S}$, et al. A new nonenzymatic method and device to obtain a fat tissue derivative highly enriched in pericyte-like elements by mild mechanical forces from human lipoaspirates. Cell Transpl. 2013;22:2063-77.

13. Dworkin RH, Turk DC, Farrar JT, Haythornthwaite JA, Jensen MP, Katz NP, et al. Core outcome measures for chronic pain clinical trials: IMMPACT recommendations. Pain. 2005;113:9-19.

14. Curtis KA, Roach KE, Applegate EB, Amar T, Benbow CS, Genecco TD, et al. Reliability and validity of the Wheelchair User's Shoulder Pain Index (WUSPI). Paraplegia. 1995;33:595-601.

15. Cleeland CS, Ryan KM. Pain assessment: global use of the Brief Pain Inventory. Ann Acad Med. 1994;23:129-38.

16. Farrar JT, Young JP Jr., LaMoreaux L, Werth JL, Poole RM. Clinical importance of changes in chronic pain intensity measured on an 11-point numerical pain rating scale. Pain. 2001;94:149-58.

17. Pentland WE, Twomey LT. Upper limb function in persons with long term paraplegia and implications for independence: Part I. Paraplegia. 1994;32:211-8.

18. Pentland WE, Twomey LT. The weight-bearing upper extremity in women with long term paraplegia. Paraplegia. 1991;29:521-30.

19. Halpern B, Chaudhury S, Rodeo SA, Hayter C, Bogner E, Potter HG, et al. Clinical and MRI outcomes after platelet-rich plasma treatment for knee osteoarthritis. Clin J Sport Med. 2013;23:238-9.

20. Shin YS, Yoon JR, Kim HS, Lee SH. Intra-articular injection of bone marrow-derived mesenchymal stem cells leading to better clinical outcomes without difference in MRI outcomes from baseline in patients with knee osteoarthritis. Knee Surg Relat Res. 2018;30:206-14.

21. Spasovski D, Spasovski V, Bascarevic Z, Stojiljkovic M, Vreca M, Andelkovic M. et al. Intra-articular injection of autologous adipose-derived mesenchymal stem cells in the treatment of knee osteoarthritis. J Gene Med. 2018;20:748-755.

22. Gill TK, Shanahan EM, Allison D, Alcorn D, Hill CL. Prevalence of abnormalities on shoulder MRI in symptomatic and asymptomatic older adults. Int J Rheum Dis. 2014;17:863-71.

23. Gonzalez-Rey E, Gonzalez MA, Varela N, O'Valle F, HernandezCortes P, Rico L, et al. Human adipose-derived mesenchymal stem cells reduce inflammatory and $\mathrm{T}$ cell responses and induce regulatory $\mathrm{T}$ cells in vitro in rheumatoid arthritis. Ann Rheum Dis. 2010;69:241-8.

24. Rha DW, Park GY, Kim YK, Kim MT, Lee SC. Comparison of the therapeutic effects of ultrasound-guided platelet-rich plasma injection and dry needling in rotator cuff disease: a randomized controlled trial. Clin Rehabil. 2013;27:113-22.

25. Mulroy SJ, Thompson L, Kemp B, Hatchett PP, Newsam CJ, Lupold DG, et al. Strengthening and optimal movements for painful shoulders (STOMPS) in chronic spinal cord injury: a randomized controlled trial. Phys Ther. 2011;91:305-24. 